\title{
The Art-Science Project: Researching the Intersection between the Visual Arts and Biological Sciences
}

\author{
Brett Jeffrey Taylor \\ College of the Arts, University of Florida
}

Faculty mentor: Lisa Iglesias, School of Art + Art History

\begin{abstract}
During the Enlightenment period, science and art were codependent disciplines. In The Art-Science Project, thirty-five painted 3D spider models were created in the Entomology and Nematology Lab at the University of Florida. The works of art encompass a diverse range of species, scales, and functions. The reproductions were designed to serve three purposes: life-size models were printed and painted to serve as decoys for reproductive research; medium scale models were distributed to international geocaches to gauge public opinion on arachnids, and large-scale models were utilized as educational tools. The created portfolio explores the area of academic art and how it can be translated into the fields of fine art and biological science. The sculptural specimens were created to understand further how art is used in scientific exploration today compared to that of antiquity. Studio art was employed as a form of research and communication. The series of thirty-five models serve different functions in that these 3D prints were utilized in mating research, public educational outreach about arachnids and teaching tools in a range of classroom settings. Simultaneously, these objects adhere to formal principles of art and can autonomously exist as artworks, thus illustrating the interdisciplinary nature of art as it relates to scientific study.
\end{abstract}

Keywords: art, science, arachnids

\section{Background}

The visual arts and biological sciences have had a complex relationship throughout antiquity and into current day. Though many artists and scientists argue that the two disciplines remain separate, others realize the codependent nature of the areas of study. In Bullot, Seely, and Davies the complex dichotomy of art and sciences is discussed by breaking the article down into perspective and opposing relationships (Bullot et al. 453). Individuals in both disciplines argue that art and science strive to achieve the same goal, answering questions and exemplifying ideas, but they depend on different principles to achieve enlightenment. Science relies on logic and the objectification of participants that take part in this conversation whereas, in the arts, intrapersonal thought can carry beyond concrete theory to think in conceptual terms.

Though the goal may be the same, some academics disagree with the codependence between the disciplines by arguing that the nature of the arts differs greatly from the sciences. There has 
been a codependence between the subjects since the conception of philosophy and the relationship between how aesthetics affect the human condition. Moreover, there is a mutualistic relationship between the two subjects allowing each to assist in the advancement of the other. This idea of dependence branches out into two opposing views when considering which discipline informs the other. Do the visual arts inform the sciences? Through illustrating scientific subject matter and condensing information down into more aesthetic, scientific summaries, the visual arts assist the sciences in condensing information into more comprehensive components. Do the sciences inform the visual arts? Thinking in the times of the Renaissance, science was used to further artistic exploration in areas such as geometry and perspective. Overall, the relationship between the visual arts and biological sciences has long been debated. This debate continues today when considering the importance of art education, the level of integration of disciplines in an interdisciplinary society, and with the introduction of new scientific and artistic technologies.

\section{Purpose}

The purpose of The Art-Science Project: Researching the Intersection between the Visual Arts and Biological Sciences, was to create a visual portfolio that explores the area of academic art and how it can be translated into the fields of fine art and biological science. The works were created within the Entomology and Nematology Department at the University of Florida to understand further how art is used in scientific exploration today compared to that of antiquity. Similar to the period of Enlightenment and in the time of antiquity, art was often created as a means of studying and researching the scientific subject matter.

During the Renaissance, artist such as Leonardo da Vinci used drawings as scientific support and to bolster scientific principles, engineering designs, and anatomical dissections. Similar to this practice, I decided to focus on creating a portfolio on a subject matter that I was not well versed in, arachnids. Through working with the Taylor Lab in the Entomology and Nematology Department at the University of Florida, I set out to see how art would facilitate understanding and could be used as an interdisciplinary method for research. Moreover, the research also looked at how the constructs of a portfolio are shifted within the setting of a lab when guided by scientific principles in addition to visual aesthetics. 


\section{Process}

The process used in this project combines the traditional methods of painting with the introduction of technologically advanced processes including scanning, photography, and threedimensional printing. The spider models are all painted from life working from small live spider specimens placed in clear dishes. In addition to the small live specimen, photographs were taken and acquired to study smaller details on a larger scale. For example, when studying the Phidippus regius female, it is difficult to study the coloration of the chelicera. The chelicera for this particular species is metallic pink, but due to its small size, the chelicera is covered by the spider's pedipalp and frontal legs. The same process is applied to the range of spider sizes from $3 \mathrm{~cm}$ to $20 \mathrm{~cm}$. The following steps communicate the process:

1. The three-dimensional spider files are acquired through the Taylor Lab at the Entomology and Nematology Department. Fellow researchers used micro-CT scanning of live spiders to create the models. These models were created during the Insects Alive project through the Entomology and Nematology Department at the University of Florida. 2. The models are printed on Fusion F306 3D printers located at the Marston Science Library at the University of Florida. The addition of plastic support material that assists in keeping the spiders form as the plastic dries and supports the spider's appendages including the legs, chelicera, and pedipalps. The support structure is printed in the same material as the spiders but in concentrated pillars. The spider form itself is printed in a hollow honeycomb-like interior structure with a thicker layer creating the contour and surface of the form.

3. The printed plastic support material that holds the spider forms stable is removed by peeling back the base layer of plastic. Once the flat layer is removed, small needle nose pliers and tweezers are used to remove the support pillars individually. Removing the material from different areas proved to be more successful in preserving the form of the spider than removing it from one region because it removed the stress in one area and distributed it amongst the entire body of the spider. When appendages did break off UV glue was used to reattach the pieces in the correct orientation. A blue UV light causes the glue to cure. This glue allowed the legs to be placed in the correct orientation before the hardening of the glue. 
4. Each model was printed in a black plastic material. A primer layer was added with the base color of the model covering the entire figure. The color was determined by studying the spider. The colors were documented in a sketchbook and then mixed in larger quantities so that the entire spider could be coated. For example, when mixing the black primer for the Phidippus regius male, phthalocyanine blue and quinacridone magenta were mixed with mars black and neutral grey to add a purple undertone to the black primer. . 5. A color blocking technique is applied next. Patterns and areas where there is a shift in color are added through defining large shapes with a solid flat color. The color applied is often a neutral mid-tone. This layer of color allows for separation between the darker primer and the local color that will be added on top of this layer. This color elevates the paint added on top when compared to the dark primer.

6. Local color and texture are added using a dry brush technique. In this process the Liquitex acrylic paint is mixed, and little paint is applied to the brush. The paint is then reduced by wiping some of the excesses off onto a sheet of paper or other material. The paint is then applied to the form and dispersed based on the orientation of the bristles. The brush is not cleaned with water in between strokes as that would result in a stroke of a solid color. The dry brush method allows colors to be layered with limited drying time in addition to giving the appearance of hair when it adheres to the texture of the threedimensional model.

7. Additional layers of color are added until the specimen resembles that of the live spider being studied. Highlights are then added to the entirety of the spider. Highlights are added to the hair, primarily at the joints, using a dry brush. Highlights are added to the eyes, chelicera, and other areas using a wet brush creating a more defined highlight. Lastly, a clear coat is applied over the entire model. The clear coat is applied in several layers to avoid fogging or clouding as the clear coat dries. The coat will allow the models to be handled more freely without damaging the paint and also reduces the amount of fading cause by exposure to light.

\section{Portfolio}

The created portfolio focused on three main principles of the scientific process, all of which were presented on a different scale. The portfolio included the following: education (large spider models), outreach (medium spider models), and research (small spider models). The portfolio 
highlighted that educational and scientific artwork conforms to certain principles that are dependent upon the intended audience. In this portfolio, the scale was significantly affected by the intended audience. The following is a breakdown of the different subsections of the portfolio and their intended purposes.

\section{Large Spider Models}

These models are primarily used as teaching tools and range from fifteen centimeters to twenty centimeters. Working on a larger scale allowed for more detailed illustrations of the anatomy, coloration, and defining characteristics of different species present in this section of the portfolio. The spiders represented within this area include Phidippus regius female, Phidippus regius male, Menemerus bivittatus male, Plexippus paykulli male, Habronattus brunneus male and Peucetia viridans. The spiders are used to educate a wide range of age groups spanning from kindergarten classrooms to college level entomology courses. The size and range of detail on these models functioned similar to an enlarged thumbnail of a scientific illustration, allowing the viewer to get an enlarged view of the specimen.

\section{Medium Spider Models}

These models will be used as outreach subjects to educate and inspire appreciation for jumping spiders. This section of the portfolio consisted of both male and female Phidippus regius spiders that are approximately five centimeters in length. These models will be placed in international geocaches starting in regions that the Taylor Lab conducts research including, Florida, Arizona, and Kenya. The models will be used to gauge the public's reaction to spiders by comparing the realistic model spiders to those that are stereotypical cartoon spiders, spiders that are distributed around the time of Halloween with the cartoon jewel eyes and bright colored hair. The lab hopes to see a more positive response to the realistic models than that of the fabricated models. In addition to the comparison, the models will include a code that leads to an educational website on the biology of jumping spiders. This will also allow researchers to observe the movement of the spider models as they are relocated. Overall, these specific models will be used to understand the public's relationship to spiders and the range to which an outreach audience is engaged.

\section{Small Spider Models}

The small life-size spider models are used in spider reproduction research. Cara McDermott, my fellow undergraduate researcher, aims to figure out if spiders react to models as they would a 
live spider. The spiders created for this section of the portfolio are to scale with the real spiders in the study and are no more than three centimeters in length. The study focuses on the Phiddippus regius. Small decoys of both males and females were created to mimic the look of this species of spider. Determining how spiders react to models in comparison to live or dead spiders could prove useful in further understanding spider communication such as courting behavior and mate selection.

\section{Conclusion}

Academic works of art are informed by the scientific subject matter and accurate representation but are achieved by following the rules of art and the principles of design. Each spider model focused upon accurately representing the subject through live observation, color studies, and preliminary sketches. Each spider, species, and gender was carefully studied before executing the models. The primary goal of studying and painting the spiders was to create a model or decoy for the real spider. This idea moves away from art for art sake, but is more informed by their function. Similar to the spiders the form follows the function.

The intended audience informs the reception of a work of academic art. For example, the spider models (Figures 1-3) would be interpreted differently in a lab setting compared to a gallery. This can also vary significantly across different age ranges. An illustration will be simplified into more elementary parts for that of a kindergarten class than for that of a college lecture. The color palette is also shifted. For illustrations intended for a younger audience, the color palate is often brighter compared to more accurate scientific renderings. Also, the function of the model greatly affected the scale to which the spider was printed, but also the amount of

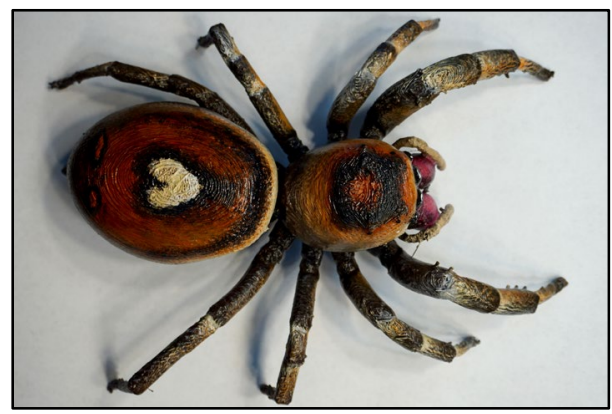

Figure 1. Teaching model

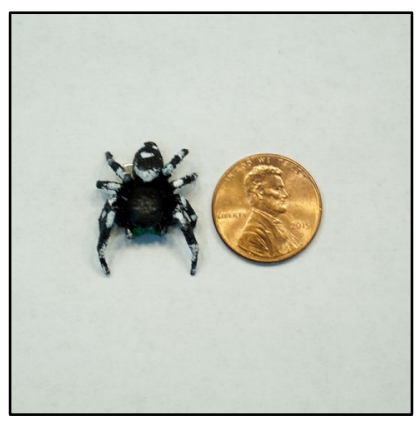

Figure 2. Research model

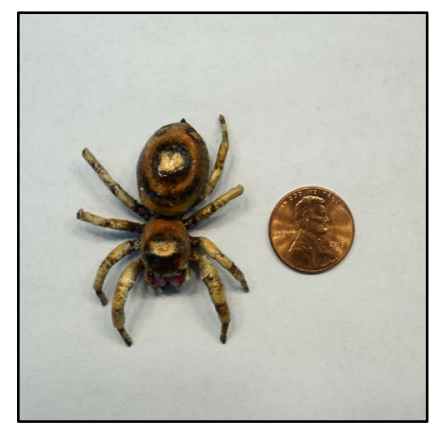

Figure 3. Outreach model detail that was required to illustrate 
the spider to the required level of realism and representation. One's understanding of the subject increases when visually representing a biological process or organism. As the artist, I have an increased understanding of the anatomy of the different spiders. Through constant visual study, it becomes clear that as one understands the structure, they can also start to understand the function of the different appendages of the organism's body. In addition, identification of different species became more evident when more reproductions of each spider were produced.

The increase of technological innovation such as three-dimensional printing has resulted in an increased relationship between the academic areas of art and science. As a result, the concept of STEM (science, technology, engineering, and math) could also include the arts. STEAM (science, technology, engineering, art, and math) would account for the codependence of the disciplines within the acronym.

\section{Testimonials}

The following is an excerpt of support from Cara McDermott a researcher that utilized the reproductive models within her research: My research focuses on behavioral studies of jumping spiders. Specifically, my research aims to determine if the species Phidippus regius will respond to lifelike, 3D printed models as if they were real spiders. Members of this species (the females in particular) have elaborate patterns all over their bodies, but their chelicera and abdomen are most notable for the beautiful coloration that helps to distinguish the species. In preparing for this project, models of spiders were 3D printed using clear resin. They then had to be painted to match the intricate designs associated with P. regius. Many studies show the importance of appearance in how jumping spiders respond to each other; therefore it was necessary to have precision in how colors and patterns were displayed on the models. I have no artistic background and was fortunate enough to have the help of Brett. Brett was able to mix different colors to match to the exact shade within certain spider's patterns. In addition, he painted many intricate displays onto less than a square inch of space to mimic the patterns that sit on the abdomen of the spiders. Additionally, the most concerning part of the model was that there may be no good way to go about mimicking the hairs that are present all over their body. Brett was able to paint them in a way that made it appear as though hairs were present even though they were not. Overall, these models were able to appear as realistic as possible due to the way they were painted. This allowed the project to proceed with few concerns about the model's appearance. 
The following is an excerpt from Dr. Lisa Taylor who runs the lab where the project was conducted: Brett's project this semester has benefited the lab in several unique ways. The first benefit was strictly practical as Brett created 35 hand-painted spider models that directly benefit our research and outreach programs. He collaborated with another undergraduate researcher to create tiny life-sized 3D models that the other student used as 'dummies' in behavioral experiments. These models are being used to understand how jumping spiders recognize and communicate with one another. The larger models that Brett created will be used in several ongoing outreach and educational initiatives - sharing our research and our appreciation for the tiny animals that inhabit our backyards is something we feel strongly about in my lab and we now have large, beautiful, and realistic hand-painted spiders to help us in this effort. In addition to the artwork that Brett created, my team of researchers has benefited from interacting with Brett and thinking broadly and creatively about the goals and processes of art and science. As biologists, we often get so focused on the specific hypotheses that we are testing, and so deep in our discussions with other biologists, which we often forget about other ways of understanding the world. Simply having Brett present and actively engaged in his own work in the lab has spurred many conversations about how the processes overlap and inform each other.

\section{References}

Bullot, Nicolas J.1, et al. "Art and Science: A Philosophical Sketch of Their Historical Complexity and Codependence." Journal of Aesthetics \& Art Criticism, vol. 75, no. 4, Fall 2017, pp. 453-463.

Doherty, M. Stephen. "Spreading the Word about Academic Realism." American Artist, vol. 70, no. 767, July/August 2006,pp.3643.

Goldstein, Carl. "Towards a Definition of Academic Art." Art Bulletin, vol. 57, no. 1, Mar. 1975, p. 102.

Marshall, Julia1,"A Systems View: The Role of Art in Education." Art Education, vol. 69, no. 3, May 2016, pp. 11-19.

Richardson, John Adkins. "Academicism and Imagination." Arts Education Policy Review, vol. 104, no. 6, July/August 2003, pp. 21-23.

Schweizer, Paul D. "John Constable, Rainbow Science, and English Color Theory." Art Bulletin, vol. 64, no. 3, Sept. 1982, p. 424.

Sotiropoulou-Zormpala, Marina1. "Seeking a Higher Level of Arts Integration across the Curriculum." Arts Education Policy Review, vol. 117, no. 1, Jan. 2016, pp. 43-54. 
Toumey, Chris1, "Technologies of Scientific Visualization." Leonardo, vol. 48, no. 1, 02 Jan. 2015, pp. 6163.

Wienroth, Matthias. "Facilitating Creative Equality in Art-Science." Leonardo, vol. 50, no. 1, Jan. 2017, pp. 42-46. 